\title{
$\mathrm{SiO}_{2} /$ 聚乙二醇非牛顿流体流变性能研究
}

\author{
伍秋美* 阮建明周忠诚黄伯云 \\ (中南大学, 粉末治金国家重点实验室, 长沙 410083)
}

\begin{abstract}
摘要 利用应力控制流变仪考察了 $\mathrm{SiO}_{2} /$ 聚乙二醇分散体系稳态和动态的流变性能. 实验结果表明, 该体系具有 剪切变稀和可逆的剪切增稠现象. 稳态应力实验中, 当应力较小时, 体系具有剪切变稀现象, 而在剪切应力 $(\sigma)$ 大 于临界剪切应力 $\left(\sigma_{c}^{s}, \sigma_{c}^{s}=9.99 \mathrm{~Pa}\right)$ 后, 体系粘度急剧增大. 在动态实验中, 剪切应力小于临界剪切应力 $\left(\sigma_{\mathrm{c}}^{\mathrm{o}}, \sigma_{\mathrm{c}}^{\circ}=15.85\right.$ Pa) 时, 储能模量 $G^{\prime}$ 减小, 耗能模量 $G^{\prime \prime}$ 与复合粘度 $\eta^{\prime \prime}$ 基本不变, 但 $\sigma>15.85 \mathrm{~Pa}$ 后, $G^{\prime} 、 G^{\prime \prime}$ 及 $\eta^{*}$ 同步增大, 且在所 研究的应力范围内, $G^{\prime \prime}$ 均大于 $G^{\prime}$. 同时还考察了测试频率、分散相含量以及分散介质平均分子量的差别对流变 性的影响. $\sigma_{\mathrm{c}}^{\mathrm{o}}$ 随测试频率的增大而变大; $\mathrm{SiO}_{2}$ 质量分数越大, $\sigma_{\mathrm{c}}^{\mathrm{o}}$ 基本不变, 但增稠现象变得更明显; 与平均分子量 小的 PEG200 体系相比, 平均分子量大的 PEG400 体系, $\sigma_{c}^{\circ}$ 并未发生改变, 但在增稠之前体系的粘度较低, 增稠 之后体系粘度增大的幅度较大.
\end{abstract}

关键词：二氧化硅，聚乙二醇，非牛顿流体，流变性，剪切增稠 中图分类号： O648, O631

\section{Study on the Rheological Properties of Silica /Polyethylene Glycol Non-Newtonian Flow}

\author{
WU, Qiu-Mei* $\quad$ RUAN, Jian-Ming ZHOU, Zhong-Cheng HUANG, Bai-Yun \\ (State Key Laboratory for Powder Metallurgy, Central South University, Changsha 410083, P. R. China)
}

\begin{abstract}
The rheological properties of dispersions of silica in polyethylene glycol (PEG) have been studied under steady and oscillation stress shear performed on AR2000 stress controlled rheometer. The system shows shear thinning and reversible shear thickening behavior. Under steady stress shear, when the stress is less than the critical stress 9.99 $\mathrm{Pa}\left(\sigma<\sigma_{\mathrm{c}}^{\mathrm{s}}\right)$, the system shows shear thinning, while, the system shows shear thickening when $\sigma>\sigma_{\mathrm{c}}^{\mathrm{s}}$. Under oscillatory stress shear, when the shear stress is less than the critical stress $15.85 \mathrm{~Pa}\left(\sigma<\sigma_{\mathrm{c}}^{\circ}\right)$ 、storage modulus $\left(G^{\prime}\right)$ decreases, loss modulus $\left(G^{\prime \prime}\right)$, and the complex viscosity $\left(\eta^{*}\right)$ almost remain unchanged; but when $\sigma>\sigma_{\mathrm{c}}^{\circ}, G^{\prime} 、 G^{\prime \prime}$ and $\eta^{*}$ increase synchronously. The magnitude of $G^{\prime \prime}$ is larger than the magnitude of $G^{\prime}$ in the range of stress studied. The higher frequency used in the experiment leads to the larger value of the critical stress. The systems almost have the same values of the $\sigma_{\mathrm{c}}^{\mathrm{o}}$ as the $\mathrm{SiO}_{2}$ mass fraction increasing, but the shear thickening phenomena are more obvious. Compared with the dispersion in PEG200, the dispersion in PEG400 shows lower complex viscosity before the critical point and a more sharp increase in the viscosity beyond the point, but has the same value of the critical stress $\left(\sigma_{\mathrm{c}}^{\circ}\right)$ and comparative viscosity at high stresses.
\end{abstract}

Keywords: Silica, Polyethylene glycol, Non-Newtonian flow, Rheological property, Shear-thickening

许多分散体系, 如药物、涂料、润滑剂等在处理 过程中都受到大的切应力作用, 这种切应力易使流
体粘度随着切应力的提高而增大, 即体系具有剪切 增稠现象. 粘度的增大使得流体的流动性变差, 会给

Received: May 26, 2005; Revised: July 27, 2005. "Correspondent, E-mail: wuqiumei20073@ sina. com; Tel: 0731-8876644. 
生产带来不利影响, 但人们利用流体的这种增稠性 能可研究设计阻尼控制设备 ${ }^{[1]}$.

目前, 已被研究的 $\mathrm{SiO}_{2}$ 分散体系主要有两大类 型. 一种是先用有机物如环己烷、3-(三甲氧基硅烷) 丙基丙烯酸脂(TPM) 对 $\mathrm{SiO}_{2}$ 进行修饰后再分散于十 八烷醇、四氢糠醇(THFFA) 等有机体系 ${ }^{[2-5]}$; 另一种是 选择亲水或疏水性的 $\mathrm{SiO}_{2}$ 直接分散于极性介质 ${ }^{[6-9]}$ (如水、水与丙酮的混合液、聚丙二醇)或非极性介 质 ${ }^{[6}$ (如无机硅油、矿物油). 文献[7-8]报道亲水性 $\mathrm{SiO}_{2}$ 的水/聚丙二醇体系具有剪切增稠现象. 文献 [10-12]报道了利用 $\mathrm{SiO}_{2}$ 的水/聚乙二醇等分散体系 的增稠性能来研究最新的液体防弹衣, 这种液体防 弹衣不仅可以减小传统防弹衣的质量, 而且可以增 加防弹衣的柔韧性, 提高强度, 但有关 $\mathrm{SiO}_{2}$ / 聚乙二 醇分散体系的流变性能目前尚未见报道. 本文主要 利用应力控制流变仪从稳态和动态方面研究了 $\mathrm{SiO}_{2} /$ 聚乙二醇分散体系的流变性能.

\section{1 实验部分}

\section{1 试 剂}

亲水性 $\mathrm{SiO}_{2}(\mathrm{~A} 200), \mathrm{BET}$ 比表面积为 $(200 \pm 25)$ $\mathrm{m}^{2} \cdot \mathrm{g}^{-1}$, 原生粒径 $12 \mathrm{~nm}$, 德国德固萨公司提供.

聚乙二醇 (polyethylene glycol, PEG), 根据平均 分子量的不同, 有PEG200和 PEG400, 二者都是低粘 度的牛顿流体, 其粘度分别为 $0.032 、 0.012 \mathrm{~Pa} \cdot \mathrm{s}$, 上 海化学试剂公司提供, 分析纯.

\section{2 实验方法}

\subsection{1 $\mathrm{SiO}_{2} / \mathrm{PEG}$ 分散体系的制备}

在 $60 \mathrm{~mL}$ 的 $\mathrm{PEG}$ 中边搅拌边加人 $\mathrm{SiO}_{2}$. 对 PEG200 体系, 分别制备 $\mathrm{SiO}_{2}$ 的质量分数为 $9 \%$ 、 $8 \% 、 5 \%$ 和 $4 \%$ 的四种 $\mathrm{SiO}_{2} / \mathrm{PEG}$ 分散体系; 对 PEG400 体系, 制备 $\mathrm{SiO}_{2}$ 质量分数为 $9 \%$ 的 $\mathrm{SiO}_{2} /$ PEG 分散体系进行对比试验, 再把样品置于 $25{ }^{\circ} \mathrm{C}$ 的 真空干燥箱中 $24 \mathrm{~h}$, 除去其中的气泡, 得到稳定的分 散体系.

\subsection{2 流变性能的测试}

在 $25{ }^{\circ} \mathrm{C}$ 恒温条件下, 分别采用稳态和动态法在 AR2000 型应力控制流变仪(美国 TA 公司)上测定 体系的流变性能. 对于 $\mathrm{SiO}_{2}$ 含量为 $9 \% 、 8 \%$ 的分散 体系, 使用雉板夹具, 雉弧度为 $2^{\circ}$, 板直径为 $40 \mathrm{~mm}$; 而对 $\mathrm{SiO}_{2}$ 含量为 $5 \%$ 、4\%的分散体系, 使用同心圆 筒夹具, 外筒内径为 $15 \mathrm{~mm}$, 内筒外径为 $14 \mathrm{~mm}$, 筒 高 $42 \mathrm{~mm}$. 使用不同的夹具对流变性能的测试没有
影响 ${ }^{[4]}$, 在我们的实验中也得到证实. 进行如下测试:

(1)稳态应力扫描, 应力范围为 $0.1 \sim 100 \mathrm{~Pa}$;

(2)频率分别为 $10 、 20 、 50 、 80 \mathrm{rad} \cdot \mathrm{s}^{-1}$ 的条件下, 进行动态应力扫描, 应力范围为 $0.1 \sim 1000 \mathrm{~Pa}$.

\section{2 结果与讨论}

\section{1 稳态流变性能}

图1为 $w\left(\mathrm{SiO}_{2}\right)=9 \%$ 的 $\mathrm{SiO}_{2} / \mathrm{PEG} 200$ 分散体系的稳 态表观粘度-剪切应力曲线. 从图 1 可看出, 在开始 施加应力时, 体系的粘度为 $6.90 \mathrm{~Pa} \cdot \mathrm{s}(\mathrm{A})$, 随着剪切 应力增大到 $1.58 \mathrm{~Pa}(\mathrm{~B})$, 粘度下降不明显, 当剪切应 力大于 $1.58 \mathrm{~Pa}$ 后, 粘度随剪切应力增大而明显下 降, 达到最低值 $1.80 \mathrm{~Pa} \cdot \mathrm{s}(\mathrm{C})$, 此后粘度随着剪切应力 的增大急剧地增大, 当剪切应力为 $50.11 \mathrm{~Pa}$, 粘度为 6.64 $\mathrm{Pa} \cdot \mathrm{s}(\mathrm{D})$ 后, 曲线基本成一平台. 粘度变化的转 折点 $\mathrm{C}$ 对应的剪切应力称为临界剪切应力 $\left(\sigma_{c}\right)$, 可 以看出该体系属于复杂的非牛顿流体.

体系粘度的变化是其微观结构及其内部基团间 相互作用的宏观表现. $\mathrm{SiO}_{2}(\mathrm{~A} 200)$ 是典型的雾化法 制得的亲水性 $\mathrm{SiO}_{2}$, 其表面具有高的自由能, 易发 生团聚, 这种团聚具有不可逆性 ${ }^{[8]} . \mathrm{SiO}_{2}$ 团聚体表面具 有大量的硅烷醇基 $(\mathrm{Si}-\mathrm{OH})$, 分散介质是极性 PEG, 分子链上有醚氧基和羟基. 当 $\mathrm{SiO}_{2}$ 粉体分散到介质 中时, $\mathrm{SiO}_{2}$ 团聚体表面的 $\mathrm{Si}-\mathrm{OH}$ 首先与 $\mathrm{PEG}$ 中 的一 $\mathrm{OH}$ 发生作用形成氢键, 在固液两相间形成网 络结构, 形成稳定的分散体系. 无外力作用的分散体 系中, 流体作用力与粒子间作用力存在着一种动态 平衡, 即 $\mathrm{SiO}_{2}$ 聚集体与 PEG 间的氢键会发生断裂, 同时这些团聚体又因粒子间作用力使得氢键重新生

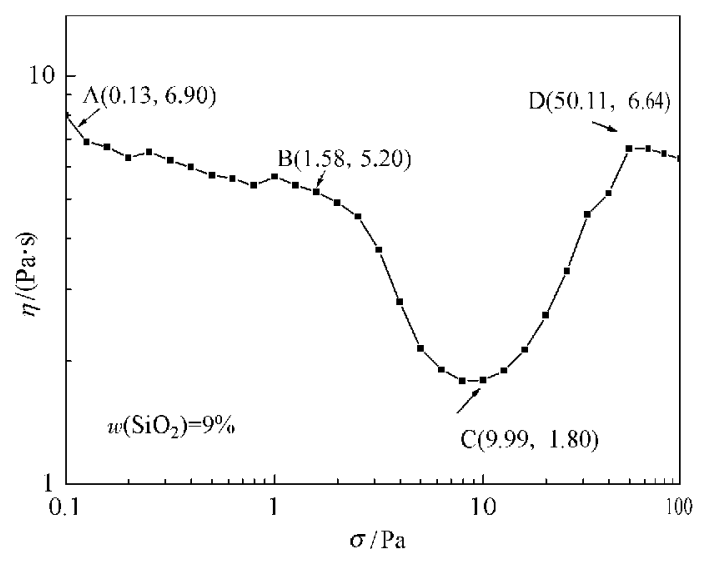

图 $1 \mathrm{SiO}_{2} / \mathrm{PEG} 200$ 分散体系的表观粘度-剪切应力曲线

Fig.1 Steady apparent viscosity as a function of shear stress for $\mathrm{SiO}_{2} /$ PEG200 dispersion 
成. 对体系施加外力时, 增大了氢键断裂的趋势, 当 外加的应力较小时, 分离的团聚体由于粒子间作用 力能很快与分散介质重新成键, 建立网状结构, 使得 体系的粘度没有发生很大的变化(图 1A 到 B). 外加 应力进一步增大, 成键的速度远不如氢键断裂的速 度, 分散相与分散介质间的氢键减少, 网状结构逐步 被破坏, 形成一些相对孤立的 $\mathrm{SiO}_{2}$ 团聚体, 粘度出 现较为明显的下降 (图 1B 到 $\mathrm{C}$ ). 当 $\sigma=\sigma_{c}^{\mathrm{s}}=9.99 \mathrm{~Pa}$ 时, 流体作用力在体系中成了主要的作用力 ${ }^{[8}$, 促使体系 中相对孤立的 $\mathrm{SiO}_{2}$ 团聚体二次团聚形成了“粒子簇”, “粒子簇” 被薄薄的介质分开, 虽然这种结合并不稳 定, 但却大大增加了流动的阻力, 使得粘度增大, 增 大至 $6.64 \mathrm{~Pa} \cdot \mathrm{s}$.

\section{2 动态流变性能}

图 2 是 $w\left(\mathrm{SiO}_{2}\right)$ 为 $9 \%$ 的 $\mathrm{SiO}_{2} / \mathrm{PEG} 200$ 分散体系, 在 频率为 $20 \mathrm{rad} \cdot \mathrm{s}^{-1}$ 时体系的储能模量 $\left(G^{\prime}\right)$ 、耗能模量 $\left(G^{\prime \prime}\right)$ 以及复合粘度 $\left(\eta^{*}\right)$ 随剪切应力的变化. 从图中可 以看出, 在所研究的剪切应力范围内, $G^{\prime \prime}>G^{\prime}$, 且当 剪切应力较低时, 即剪切应力小于 $15.85 \mathrm{~Pa}\left(\sigma<\sigma_{\mathrm{c}}^{\circ}\right)$, $G^{\prime \prime}$ 以及 $\eta^{*}$ 只有轻微的降低, 而 $G^{\prime}$ 却明显地下降, 当 $\sigma=\sigma_{\mathrm{c}}(15.85 \mathrm{~Pa})$ 时, $G^{\prime}$ 和 $G^{\prime \prime}$ 以及 $\eta^{*}$ 达到一个最低值, 随后随着剪切应力的增大都达到最大值, 且最大值 都大于体系的起始值.

粘弹性理论认为 ${ }^{[13]}$, 弹性是体系的固体行为, 粘 性为体系的液体行为, 可分别用储能模量 $\left(G^{\prime}\right)$ 和耗 能模量 $\left(G^{\prime \prime}\right)$ 之值表示体系的弹性和粘性的强度; 而 $\eta$ *是物质对动态剪切总阻抗的量度, 它是由储能粘 度(弹性成分)和动态粘度(粘性成分)两部分构成. 当

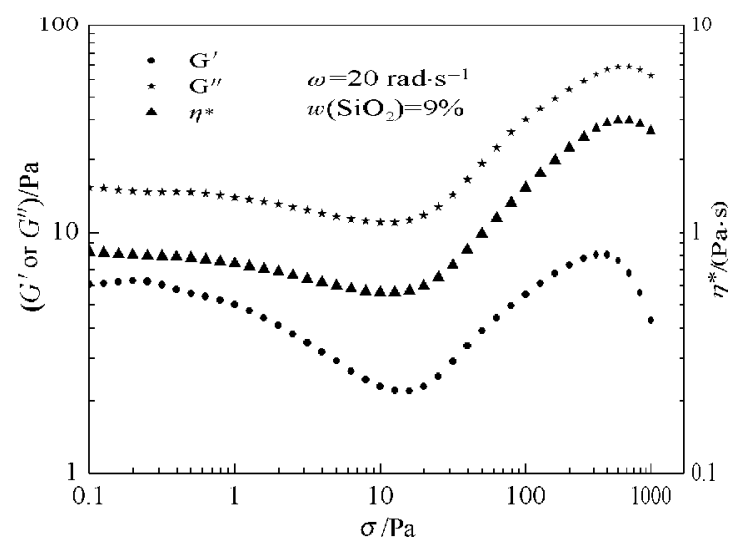

图 2 储能模量 $\left(G^{\prime}\right)$ 、耗能模量 $\left(G^{\prime \prime}\right)$ 以及复合粘度 $\left(\boldsymbol{\eta}^{*}\right)$ 与动 态剪切应力 $(\sigma)$ 的关系

Fig.2 The storage modulus $\left(G^{\prime}\right)$, loss modulus $\left(G^{\prime \prime}\right)$ and $\operatorname{complex} \operatorname{viscosity}\left(\eta^{*}\right)$ as a function of oscillation stress $(\sigma)$ for $\mathrm{SiO}_{2} /$ PEG200 dispersion
$0.10 \mathrm{~Pa}<\sigma<15.85 \mathrm{~Pa}$ 时, 体系中固液间的网状结构 逐步被破坏, $G^{\prime}$ 逐渐减少, $G^{\prime \prime}$ 和 $\eta^{*}$ 基本不变, 可以看 出 $\eta^{*}$ 主要由粘性成分构成. 在 $\sigma>\sigma_{\mathrm{c}}^{\circ}(15.85 \mathrm{~Pa})$ 后, “粒子簇”的形成使得 $G^{\prime}$ 和 $G^{\prime \prime}$ 以及 $\eta^{*}$ 同步增大.

图 3 为 $w\left(\mathrm{SiO}_{2}\right)=9 \%$ 的 $\mathrm{SiO}_{2} / \mathrm{PEG} 200$ 分散体系剪 切增稠的可逆性曲线. 图中, 实心点曲线代表应力从 $0.10 \mathrm{~Pa}$ 增大到 $1000 \mathrm{~Pa}$ 的扫描, 空心点线为应力从 $1000 \mathrm{~Pa}$ 减小到 $0.10 \mathrm{~Pa}$ 的扫描, 频率都恒定为 20 $\mathrm{rad} \cdot \mathrm{s}^{-1}$. 从图中可以看出, 这两条曲线基本重合, 尤其 在应力小于 $100 \mathrm{~Pa}$ 的情况下, 重合性非常好, 表明 该体系的增稠现象具有可逆性. 进一步可以得出, 由 于流体作用力而生成的 “粒子簇” 并不很稳定, 随着 外力的减小, 形成的 “粒子簇” 将重新分解为 $\mathrm{SiO}_{2}$ 的 一次团聚体, 分散到介质中.

图4为 $w\left(\mathrm{SiO}_{2}\right)=9 \%$ 的 $\mathrm{SiO}_{2} / \mathrm{PEG} 200$ 分散体系, 在 不同的频率条件下的动态复合粘度-应力曲线. 从 图中可见, 随着频率从 $10 \mathrm{rad} \cdot \mathrm{s}^{-1}$ 增大到 $80 \mathrm{rad} \cdot \mathrm{s}^{-1}$, 临 界增稠点 $\left(\sigma_{c}^{\circ}, \eta^{*}\right)$ 分别为 $\mathrm{A}(6.26,0.74) 、 \mathrm{~B}(12.88,0.56)$ 、 $\mathrm{C}(61.96,0.44) 、 \mathrm{D}(167.11,0.37)$, 增稠点对应的 $\sigma_{\mathrm{c}}^{\circ}$ 增 大, $\eta^{*}$ 减小. 且在所研究的应力范围内, 频率较小时 测得的复合粘度较大, 剪切增稠前粘度下降得更明 显, 其原因可能是在相同应力条件下, 频率较大时, 粒子与分散介质间的氢键断裂较快, 体系粘度较小, 同时体系中生成的相对孤立的团聚体也分散得更 广, 形成“粒子簇”的几率就小, 因此要在更大的应力 条件下才能发生增稠现象.

\section{3 分散相含量对增稠性能的影响}

图5为不同 $w\left(\mathrm{SiO}_{2}\right)$ 分散体系的动态复合粘度-

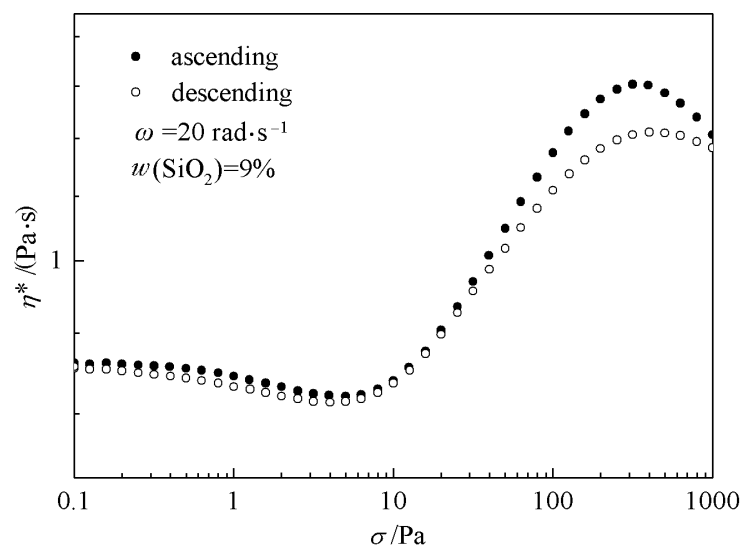

图 $3 \mathrm{SiO}_{2} /$ PEG200 分散体系剪切增稠的可逆性曲线

Fig.3 Reversible shear thickening behavior for $\mathrm{SiO}_{2} /$ PEG200 dispersion 


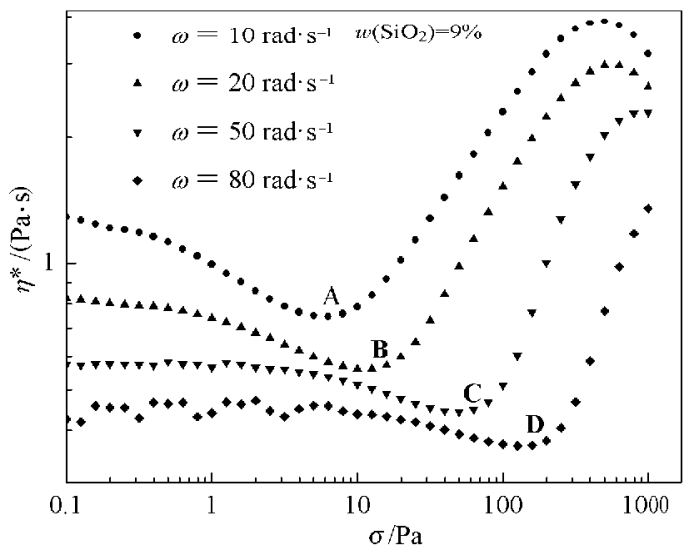

图 4 不同频率的动态复合粘度-应力曲线

Fig.4 Complex viscosity as function of oscillation stress for $\mathrm{SiO}_{2} /$ PEG200 dispersions

应力曲线. 从图中可以看出, 随着 $\mathrm{SiO}_{2}$ 含量的减少, $\sigma_{\mathrm{c}}^{\mathrm{o}}$ 基本不变, 大致为 $100 \mathrm{~Pa}$, 但增稠现象越来越不明 显, 且在研究范围内, 固相含量少的体系粘度较小. 这主要是由于体系中固体粒子的减少导致了固液间 的作用力变弱, 粘度降低, 在剪切增稠后, 形成的粒 子簇也减少或更小, 对流动的阻力也就减少, 所以增 稠效果就不明显.

\section{4 分散介质对增稠性能的影响}

图 6 为 PEG 的平均分子量对剪切增稠的影响. 图中实心点为 PEG200 的体系, 空心点为 PEG400 的体系. 比较频率为 $20 \mathrm{rad} \cdot \mathrm{s}^{-1}$ 的两条曲线, 可看出 在相同频率的条件下, 两体系增稠点分别为 $\mathrm{A}$ (12.59, 0.56)、 $\mathrm{A}^{\prime}(12.59,0.20)$, 临界增稠点对应的应 力不变, 只是 PEG400 的体系对应的粘度较小, 且在 整个研究应力范围内, 在增稠点之前, PEG400 体系

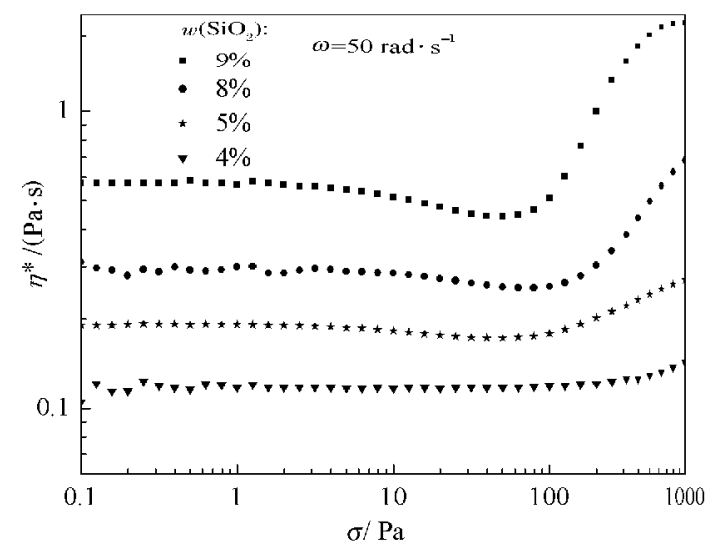

图 5 不同 $\mathrm{SiO}_{2}$ 浓度分散体系的动态复合粘度-应力曲线

Fig.5 Complex viscosity as function of oscillation stress for $\mathrm{SiO}_{2} /$ PEG200 dispersions containing different masses of $\mathrm{SiO}_{2}$

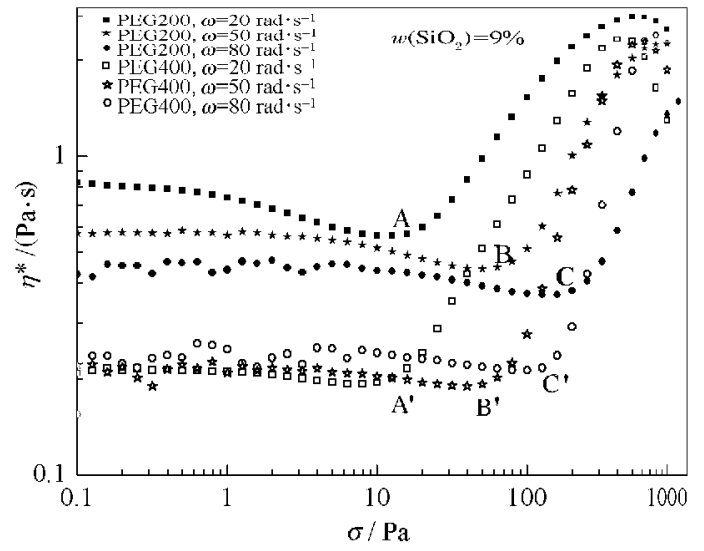

图 6 不同分散介质体系的复合粘度-应力曲线

Fig.6 Complex viscosity as function of oscillation stress for dispersions in different PEG

的粘度都比 PEG200 的体系低, 但在增稠点之后, PEG400 体系粘度增大的幅度大于 PEG200 体系, 最 终粘度接近 PEG200体系. 频率为 $50 、 80 \mathrm{rad} \cdot \mathrm{s}^{-1}$ 时 PEG200、PEG400 两个体系粘度随应力的变化曲线 与频率为 $20 \mathrm{rad} \cdot \mathrm{s}^{-1}$ 时类似, 只是频率分别为 $50 、 80$ $\mathrm{rad} \cdot \mathrm{s}^{-1}$ 时, PEG400 体系的最终粘度分别是等于、大 于 PEG200 的最终粘度. 对于相同质量的 PEG 而言, 平均分子量大的 PEG400 分子链长, 含有的羟基较 少, 与二氧化硅团聚体形成的氢键数也较少, 这样固 液间的作用力弱, 粘度就小些; 在增稠之后, 粘度的 增大主要是由于 “粒子簇” 的生成, 体系中含有的 $\mathrm{SiO}_{2}$ 的量是一样的, 对 PEG400 体系来说, 固液间的 作用力较弱, 更有利于 $\mathrm{SiO}_{2}$ 摆脱液相束缚参与 “粒 子簇”的生成, 所以增稠之后, 粘度增大的幅度大些, 而且频率越大, 增稠之后两体系粘度增大幅度的差 别也越大.

\section{3 结 论}

1) $\mathrm{SiO}_{2} / \mathrm{PEG}$ 分散体系具有剪切变稀和可逆的 剪切增稠现象. 在增稠之前, 即 $\sigma<\sigma_{\mathrm{c}}$, 应力的增加 破坏了分散相与分散介质之间的作用力, 使得分散 相在体系中形成自由体, 体系粘度下降; 在体系增 稠之后, 即 $\sigma>\sigma_{\mathrm{c}}$, 流体作用力成了主要的作用力, 促使了分散相 “粒子簇” 的形成, 使得体系的粘度增 大, 但这种 “粒子笶”的稳定性差, 当应力减小时, “粒 子簇” 重新分解分散到介质中, 又与介质形成稳定的 结构.

2) 当 $\sigma<\sigma_{\mathrm{c}}^{\circ}$ 时, $G^{\prime \prime}$ 只有轻微的降低, 而 $G^{\prime}$ 却有 明显的下降, 当 $\sigma=\sigma_{\mathrm{c}}^{\circ}(15.85 \mathrm{~Pa})$ 时, $G^{\prime}$ 和 $G^{\prime \prime}$ 达到一个 
最低值后随着 $\sigma$ 的增大而增大, 且在所研究的 $\sigma$ 范围 内, $G^{\prime \prime}$ 大于 $G^{\prime}$.

3) 随着 $\mathrm{SiO}_{2}$ 质量分数的增大, 体系粘度增大, $\sigma_{\mathrm{c}}^{\circ}$ 基本不变, 但增稠效果更明显; 对于平均分子量不 同的 PEG 体系, 体系的 $\sigma_{\mathrm{c}}^{\circ}$ 基本没有改变, 但分子量 较大的 PEG400 体系在增稠之前的粘度较低, 增稠 之后与分子量较小的 PEG200 体系的粘度相当. 这 主要与体系中固液两相形成氢键的多少以及增稠后 形成的“粒子簇”的大小和多少有关.

\section{References}

1 Suciu, C. V.; Iwatsubo, T.; Deki, S. Journal of Colloid and Interface Science, 2003, 259: 62

2 Jonathan, B.; Wagner, N. J. Journal of Rheology, 1996, 40: 899

3 Fritz, G. ; Maranzano, B. J.; Wagner, N. J. Journal of Non-Newtonian Fluid Mechanism, 2002, 102: 149
4 Maranzano, B. J.; Wagner, N. J. Journal of Chemical Physics, 2001, 114: 10514

5 Marazano, B. J.; Wagner, N. J. Journal of Rheology, 2001, 45: 1205

6 Raghavan, S. R.; Khan, S. A. Journal of Rheology, 1995, 39: 1311

7 Lee, Y. S.; Wagner, N. J. Rheology Acta, 2003, 42: 199

8 Raghavan, S. R.; Khan, S. A. Journal of Colloid and Interface Science, 1997, 85: 57

9 Toshiyuki, S. Journal of Rheology, 1994, 38: 601

10 Tan, V. B. C.; Tay, T. E.; Teo, W. K. Solids and Structures, 2005, 42: 1561

11 Lee, B. L.; Walsh, T. F.; Won, S. T.; Patt, H. M. Journal of Composite Materials, 2001, 35: 1605

12 Lee, Y. S.; Wetzel, E. D.; Wagner, N. J. Journal of Materials Science, 2003, 38: 2825

13 Schramm, G. A. Practical approach to rheology and rheometry. Trans. Li, X. J. Beijing: Petroleum Industry Press, 1998: 113 [实用流变测量学. 李晓军译. 北京: 石油工业出版社, 1998: 113]

\section{第四届东方胶化杯全国胶体化学研究生优秀成果奖评选通知}

为促进中国的胶体化学发展,鼓励这一领域青年学生的进取精神, 2002 年起特设立全国胶体化学研究生奖学金,以表彰优 秀的胶体化学专业研究生.

\section{申请资格与范围:}

全国范围内各大学、科研机构在读的胶体化学专业研究生(含博士生)均有资格申请. 申请人应在胶体与界面化学领域取得 较为突出的创新性结果. 发表一定数量的优秀科研论文(含已接受). 申请人的所有成果必须是在研究生阶段进行或完成的. 在职研 究生不参与本奖学金评选.

\section{评选程序:}

1) 本人申请: 申请人须提交个人简历(含联系方式)、学术论文发表及已接受目录(含全部作者的正确顺序、期刊、年、卷(期)、 页), 科研工作自评, 论文工作概况. 已接受文章须具备期刊的接收函(含 E-mail).

2) 单位推荐: 申请人须有本单位两名副教授以上职称的老师(含导师)推荐.

3) 专家评审: 由中国化学会物理化学学科委员会胶体与界面化学学科组组织专家进行评审, 确定获奖名单.

\section{评选进程:}

2006 年 1 月 31 日前递交个人申请与推荐材料(含电子版), 经专家评审后于 2006 年 3 月正式公布并举行颁奖仪式.

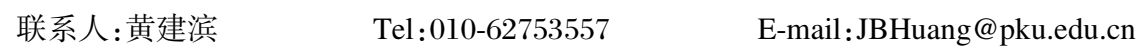

邮寄地址: 北京大学化学与分子工程学院 100871

本奖学金的设立获得了北京东方德菲胶体化学仪器有限公司的大力支持与独家赞助.

本奖学金评选工作的最终解释权在中国化学会物理化学学科委员会胶体与界面化学学科组.

全国胶体与界面化学学科组 2005 年 11 月 